\title{
A rare cause of ureteric stricture and hydronephrosis: metastatic esophageal cancer to the urinary bladder
}

\author{
Darren Goh, Xin Ling Teo, Sey Kiat Terence Lim \\ Department of Urology, Changi General Hospital, Singapore 529889, Singapore.
}

Correspondence to: Dr. Darren Goh, Department of Urology, Changi General Hospital, 2 Simei Street 3, Singapore 529889, Singapore. E-mail: darren.goh.w.y@singhealth.com

\begin{abstract}
How to cite this article: Goh D, Teo XL, Lim SKT. A rare cause of ureteric stricture and hydronephrosis: metastatic esophageal cancer to the urinary bladder. Mini-invasive Surg 2019;3:17. http://dx.doi.org/10.20517/2574-1225.2019.06
\end{abstract}

Received: 12 Feb 2019 First Decision: 4 May 2019 Revised: 15 May 2019 Accepted: 17 May 2019 Published: 18 Jun 2019

Science Editor: Giulio Belli Copy Editor: Cai-Hong Wang Production Editor: Huan-Liang Wu

\begin{abstract}
The presence of hydronephrosis usually signifies the presence of significant urinary tract obstruction, more commonly at the level of the ureter, and occasionally at the bladder outlet in cases of bilateral hydronephrosis. Unilateral hydronephrosis is most commonly caused by a ureteric stone or stricture, and rarely caused by neoplasm. Metastatic disease to the urinary bladder is rare and usually presents with hematuria, and we report the first case of hydronephrosis resulting from a metastatic esophageal cancer to the bladder.
\end{abstract}

Keywords: Hydronephrosis, metastatic esophageal cancer, ureteric stricture

\section{INTRODUCTION}

Unilateral hydronephrosis is most commonly caused by obstruction of the ureter due to the presence of a ureteric stone or stricture, and rarely secondary to a primary ureteric or bladder neoplasm or from direct invasion or external compression by locally advanced cancers from adjacent organs such as the lower gastrointestinal and female genitourinary tract. We report the first case of a patient presenting with a ureteric stricture with hydronephrosis and acute kidney injury secondary to a metastatic esophageal cancer to the urinary bladder.

\section{CASE REPORT}

This is a 72-year-old Chinese male who was diagnosed with cardio-esophageal cancer on esophago-gastroduodenoscopy for work up of dyspepsia. Biopsies revealed poorly differentiated adenocarcinoma. Staging

\footnotetext{
(c) (7) (c) The Author(s) 2019. Open Access This article is licensed under a Creative Commons Attribution 4.0 International License (https://creativecommons.org/licenses/by/4.0/), which permits unrestricted use sharing, adaptation, distribution and reproduction in any medium or format, for any purpose, even commercially, as long as you give appropriate credit to the original author(s) and the source, provide a link to the Creative Commons license, and indicate if changes were made.
}

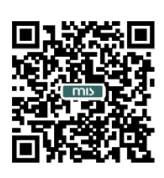


computed tomography (CT) scan of the chest, abdomen and pelvis showed a heterogeneously enhancing mural thickening of the lower esophagus, with no enlarged regional lymph nodes and a fluorodeoxyglucose positron emission tomography (FDG PET) scan did not show any nodal or distant metastasis. He underwent neoadjuvant chemotherapy and radiotherapy, followed by Ivor Lewis esophagectomy, total gastrectomy and colonic interposition. Final histology showed invasive poorly differentiated adenocarcinoma at the gastro-esophageal junction, arising in the background of Barrett's esophagus. The tumor invaded the periesophageal adventitia and involved the serosa of stomach with extensive peri-neural invasion seen. All 45 lymph nodes were negative for malignancy and the final staging was pT4aNoMo with clear resection margins. His case was discussed at the gastrointestinal tumor board and decision was for surveillance alone, without a need for adjuvant chemotherapy or radiotherapy. He was followed up with surveillance CT scan of the abdomen and pelvis and was noted to have new onset moderate left hydronephrosis 9 months after the operation. The left hydronephrosis extended all the way down to the urinary bladder with no obvious cause of obstruction or lesions noted. He underwent a ureteroscopy and was noted to have a $4-\mathrm{cm}$ tight distal ureteric stricture with unhealthy looking ureteric mucosa, but no obvious bladder or ureteric lesion [Figure 1A]. Balloon dilatation of the ureteric stricture was performed and a double-J stent was inserted, which was removed 2 weeks later. He was planned for follow up CT intravenous pyelogram to evaluate the ureteric stricture but was subsequently noted to develop acute kidney injury with a rise in serum creatinine. A noncontrast CT scan of the abdomen and pelvis now showed an area of bladder wall thickening in the region of the left ureteric orifice with worsening hydronephrosis [Figure $1 \mathrm{~B}$ ]. He underwent a rigid cystoscopy and was noted to have edematous bladder wall mucosa with solid looking areas and had a transurethral resection of bladder tumor performed; histology showed normal urothelium with submucosal infiltration by metastatic adenocarcinoma with signet cell morphology. Immunostains show the carcinoma cells staining positively with CK7 and CK20, and negatively with CDX2, TTF1, Napsin A, S100, GATA3, PSA and PSAP. These findings were in keeping with metastatic poorly differentiated adenocarcinoma of esophagus/gastric origin. A re-staging FDG PET CT scan was performed and did not show any obvious nodal or distant metastasis. He was referred to the medical oncologist for palliative treatment of the metastatic esophageal cancer.

\section{DISCUSSION}

Esophageal cancer is the eighth most common cancer worldwide and esophageal cancer most commonly metastasize to the liver and peritoneum, regional lymph nodes, lung, stomach, kidney, adrenals and bone $^{[1]}$. Esophageal cancer can metastasize via several different routes; with direct invasion, lymphatic and hematogenous spread being the more common routes of spread. Other mechanisms of metastasis include trans-peritoneal and intra-luminal implantation. The most common sites of esophageal metastases include liver, regional lymph nodes and lung, but unexpected sites of metastasis have increasingly been reported. It is unclear how esophageal cancer can spread to the urinary bladder, but one possibility may be via the hematogenous route.

Shaheen et al. ${ }^{[2]}$ performed a systematic review on esophageal cancer metastases to unexpected sites and found 164 cases reported, of which there were 14 cases of metastatic spread to the urinary tract (10 to kidney, 2 to penis and 2 to testis/spermatic cord), but none was found to have spread to the urinary bladder.

Metastatic spread to the bladder constitutes $2 \%$ of all bladder neoplasms, and most commonly they occur by direct invasion rather than from distant spread ${ }^{[3]}$. Velcheti and Govindan ${ }^{[4]}$ reviewed 264 cases of metastatic disease to the bladder and found the most common primary site to be genitourinary and colorectal; and melanoma, breast and stomach are the commonest primary foci for distant spread.

The urinary bladder is an extremely rare site of metastasis from the esophagus with less than 5 cases reported worldwide. An extensive literature search found only four reported cases of metastatic disease to 

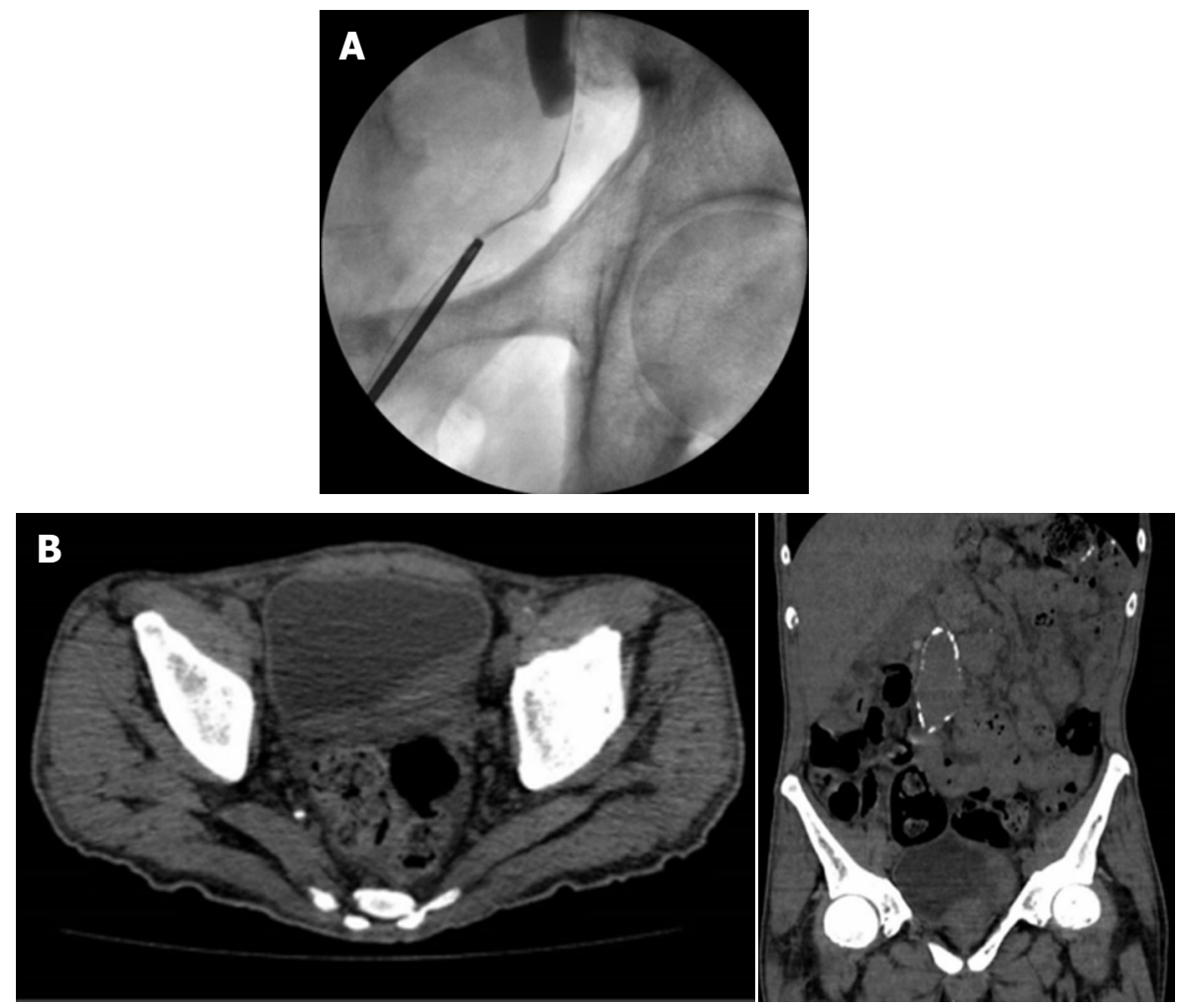

Figure 1. This (A) shows the initial intra-operative contrast study which demonstrates the left ureteric stricture with hydronephrosis. Follow up non contrast scan (B) of the abdomen and pelvis which shows left bladder wall thickening with worsening hydronephrosis

the bladder originating from the esophagus, with gross hematuria being the main presenting symptom. This is the first known reported case of a metastatic esophageal cancer to the bladder presenting with hydronephrosis and acute kidney injury.

Matsumoto et al. ${ }^{[5]}$ in 2004 described a patient who presented with hematuria and was found to have metastatic intra-pelvic tumour from oesophageal cancer which invaded the bladder, rectum and sigmoid colon. Hargunani et al. ${ }^{[6]}$ in 2005 reported a patient with esophageal cancer who underwent curative resection after neoadjuvant chemotherapy; but was subsequently found to have metastasis to the bladder after he presented with gross hematuria. Schuurman et al. ${ }^{[7]}$ reported another case of metastatic esophageal cancer to the bladder in 2009. This was diagnosed as part of staging of the esophageal cancer and was treated with palliative radiotherapy. Katz et al ${ }^{[8]}$ in 2017 reported a patient with esophageal cancer and staging scan showed metastatic liver lesions with retroperitoneal adenopathy. She completed chemotherapy, but developed anaemia with hematuria and was subsequently found to have metastatic esophageal cancer to the bladder. Two of these cases were diagnosed at presentation to have synchronous metastasis to the bladder while the other 2 cases developed metachronous metastasis to the bladder on follow up. Most of the cases were treated with palliative intent, and only one case underwent operation with curative intent, only to develop metachronous metastasis to the bladder subsequently. Our case is the second reported case to be diagnosed with metachronous metastasis to the bladder after treatment of esophageal cancer with curative intent.

The two main subtypes of esophageal carcinoma are adenocarcinoma and squamous cell carcinoma (SCC). There have been studies that looked at patterns of metastasis based on the histological subtype, and it has been found that esophageal adenocarcinoma is more likely to develop liver and brain metastasis; while SCC tend to develop lung metastasis, with no difference found for lymph node and bone metastases. Esophageal 
metastasis to the urinary bladder and its association with the histological subtype has not been reported in view of its rare occurrence, and will not make for a meaningful analysis in view of its small numbers.

This is the first reported case of a ureteric stricture with hydronephrosis and acute kidney injury resulting from a metachronous esophageal cancer to the bladder. An accurate diagnosis of the cause of hydronephrosis is crucial in all cases as it can affect the management of patients. In this case, the metastatic disease had infiltrated the bladder submucosa and initial cystoscopy and ureteroscopy did not show any obvious bladder mucosal or ureteric lesion, and he was treated as for a benign ureteric stricture. The initial CT scan did not show any other abnormality such as local recurrence, metastatic spread to other organs or enlarged lymph nodes to suggest metastatic disease. This case illustrates the importance of taking into consideration patient's past medical history and to consider the various differentials for hydronephrosis, regardless of how unlikely or rare the possibility is. Ureteric re-implantation would be one of the treatment options for a long segment tight ureteric stricture or if the stricture had recurred after balloon dilatation. A decision was made to follow up with imaging first, which demonstrated the development of bladder wall thickening which eventually led to the diagnosis of a metastatic esophageal cancer. Treatment will now be with palliative intent and a percutaneous nephrostomy has been inserted to relieve the hydronephrosis, and the patient will be planned for palliative chemotherapy. His baseline renal function was initially normal, but he subsequently developed acute kidney injury due to obstructive uropathy. The percutaneous left nephrostomy relieved the obstruction and his renal function returned to normal. A normal renal function is important as many chemotherapeutic agents may cause nephrotoxicity and cannot be given in patients with renal impairment, or may need renal dose adjustment. For this patient, he was treated with capecitabine (Xeloda).

\section{DECLARATIONS}

\section{Authors' contributions}

Literature review: Goh D

Creation of manuscript: Goh D, Teo XL, Lim SKT

\section{Availability of data and materials}

Not applicable.

\section{Financial support and sponsorship}

None.

\section{Conflicts of interest}

All authors declared that there are no conflicts of interest.

\section{Ethical approval and consent to participate}

Informed consent to participate in the study was obtained from the patient.

\section{Consent for publication}

Written informed consent was obtained from the patient for publication.

\section{Copyright}

(c) The Author(s) 2019.

\section{REFERENCES}

1. Napier KJ, Scheerer M, Misra S. Esophageal cancer: a review of epidemiology, pathogenesis, staging workup and treatment modalities. World J Gastrointest Oncol 2014;6:12-20. 
2. Shaheen O, Ghibour A, Alsaid B. Esophageal cancer metastases to unexpected sites: a systematic review. Gastroenterol Res Pract 2017;2017:1657310.

3. Bates AW, Baithun SI. Secondary neoplasms of the bladder are histological mimics of nontransitional cell primary tumours: clinicopathological and histological features of 282 cases. Histopathology 2000;36:32-40.

4. Velcheti V, Govindan R. Metastatic cancer involving bladder: a review. Can J Urol 2007;14:3443-8.

5. Matsumoto Y, Mibu H, Kagebayashi Y, and Miyasaka Y. Metastatic intrapelvic tumor from esophageal cancer: a case report. Hinyokika Kiyo 2004;50:725-7.

6. Hargunani R, Al-Dujaily S, Abdulla AKS, Osborne DR. Haematuria as a presentation of metastatic oesophageal carcinoma. Int Semin Surg Oncol 2005;2:4.

7. Schuurman JP, De Vries Reilingh TS, Roothaan SM, Bijleveld RT, Wiezer MJ. Urinary bladder metastasis from an esophageal adenocarcinoma: a case report. Am J Gastroenterol 2009;104:1603-4.

8. Katz H, Saad RE, Denning K, Pacioles TO. A rare case of oesophageal adenocarcinoma with urinary bladder metastasis. Case Rep Gastrointest Med 2017;2017:9343896. 